\title{
Visualization of Phase Segregation and Surface Reconstruction of Pt-Based Bi- metallic Clusters During In Situ Oxidation
}

\author{
Jane Howe, ${ }^{1,2}$, Ya-Huei (Cathy) Chin, ${ }^{3}$ Weifeng Tu, ${ }^{3}$ Yifei Yang, ${ }^{3}$ Junnan Shangguan, ${ }^{3}$ Stas Dogel ${ }^{1}$, \\ David Hoyle ${ }^{1}$, Matthew Reynolds ${ }^{4}$, Hooman Hosseinkhannazer ${ }^{4}$, and Doug Perovic ${ }^{2}$ \\ 1. Hitachi High-Technologies Canada Inc, Toronto, Canada. \\ 2. Department of Materials Science and Engineering, University of Toronto, Canada. \\ 3. Department of Chemical Engineering and Applied Chemistry, University of Toronto, Canada. \\ 4. Norcada Inc, Edmonton, Canada.
}

Bi-metallic clusters such as Ni-Au [1], Pt-Ni [2], Pd-Au [3], Pt-Pd [4], and Pt-Au of nano-meter dimensions are effective catalysts for hydrocarbon activation (Ni-Au [1], Pt-Ni [2]), alcohol oxidation $(\mathrm{Pd}-\mathrm{Au})$ [3], aromatic hydrogenation (Pt-Pd) [4], and water-gas-shift (Pt-Au) [5] reactions. Their catalytic performance is dictated predominantly by the number and type of sites at their surfaces and thus by their size, shape, and local atomic ensembles at cluster surfaces. When exposed to reaction mixtures, these clusters may undergo dynamics reconstruction at surfaces and in the bulk phase, driven by changing chemical potentials. As an example, bi-elemental alloy with a more oxophilic metal may disintegrate and undergo bulk phase segregation during oxidation reactions, as oxygen atoms solvate into the cluster bulk and the more oxophilic metal undergoes bulk oxidation.

Herein, we study the phase segregation and surface reconstruction of Pt-M bimetallic clusters $(\mathrm{M}=\mathrm{Ru}$, Pd) via in situ electron microscopy with a Hitachi heating holder with Norcada MEMs-based heating chips. Using a Hitachi HF-3300 environmental TEM/STEM, we carried out in situ oxidation studies of these bi-metallic clusters at $1 \mathrm{~Pa}_{2}$ up to $400{ }^{\circ} \mathrm{C}$. As a comparison, in situ heating in vacuum was also carried out with an aberration-corrected Hitachi HD-2700 STEM. Both instruments are equipped with a secondary electron detector, which can reveal the surface features via secondary electron (SE) imaging, while simultaneously probe the clusters through its entire thickness (bulk) with transmitted electrons [6]. This ability is advantageous in studying a heterogeneous catalyst material, because it permits the selective visualization of bulk atoms and surface ones, beyond a traditional two-dimensional projection in TEM [7].

In situ and ex situ STEM revealed distinctive stages of change in these nanometer sized clusters and especially, the oxygen solvation and oxide shell formation on the Pt-M clusters in response to an increase in oxygen chemical potentials. For the case of as-prepared Pt-Ru clusters, uniform contrast of the ADF and SE images (Fig. 1) and even distribution of Pt-Ru EDS mapping suggested that Pt-Ru formed a solid solution on the surface and through the bulk. During oxidation in $1 \mathrm{~Pa} \mathrm{O}_{2}$ at $350{ }^{\circ} \mathrm{C}$, $\mathrm{Ru}$ as the oxophilic metal preferentially migrated to the shell of the Pt-Ru clusters and formed an oxide layer, but a small portion of $\mathrm{Pt}$ remained at the surfaces as the active site during catalysis. As the oxygen dissolution step occurred, the clusters changed their overall shape and size. At the final stage of the oxidation, some of the $\mathrm{Ru}$ completely segregated from $\mathrm{Pt}$ and formed ruthenium oxides adjacent to the original crystallite (Fig. 2). And finally, the ruthenium oxide became amorphous.

These case studies, through combined kinetic, isotopic, and in-situ secondary and transmitted electron imaging techniques, show the generation of reactive oxygen centers, formed only during reactions when incorporating an oxophilic metal onto the Pt clusters. The findings from this work may pave the way towards designing catalysts through tailoring the chemical composition for optimal catalytic performance [8]. 


\section{References:}

[1] Y-H Chin et al, J. Catal. 244 (2006) 153.

[2] S Ozkara-Aydinoglu, and A E Aksoylu, Int. J. Hydrogen Energ. 36 (2011) 2950.

[3] N Dimitratos et al, J. Catal. 244 (2006) 113.

[4] S Albertazzi, et al, J. Catal. 223 (2004) 372.

[5] M M Mohamed and K S Khairou, J. Colloid Inter. Sci. 354 (2011) 100.

[6] Y Zhu, et al, Nature Mater. 8 (2009) 808.

[7] J Y Howe et al, Nanoscale Res. Lett. 9 (2014) 614.

[8] Electron microscopic studies were carried out at the Ontario Centre for the Characterisation of Advanced Materials (OCCAM), University of Toronto.

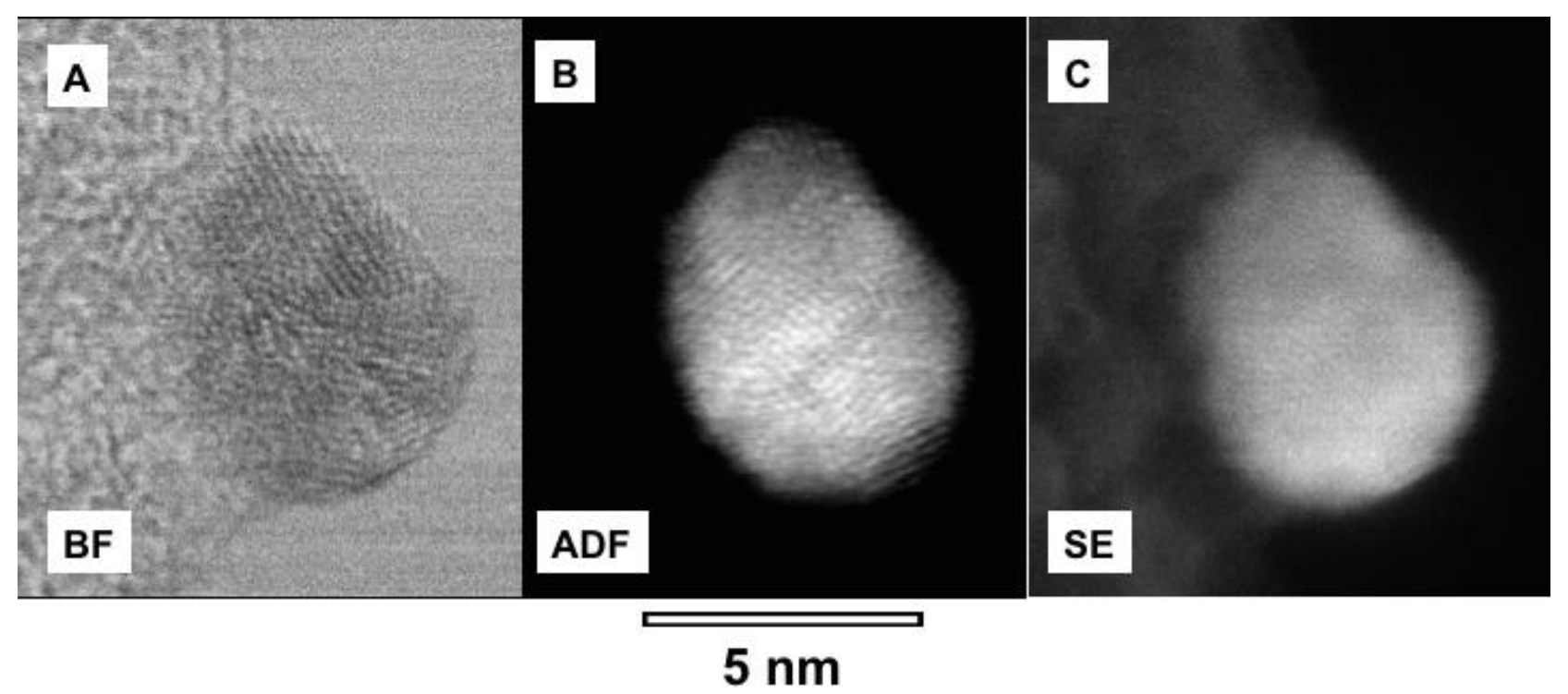

Fig. 1. Pt-Ru nanoclusters prior to the in situ study, reveal random (alloyed) composition.
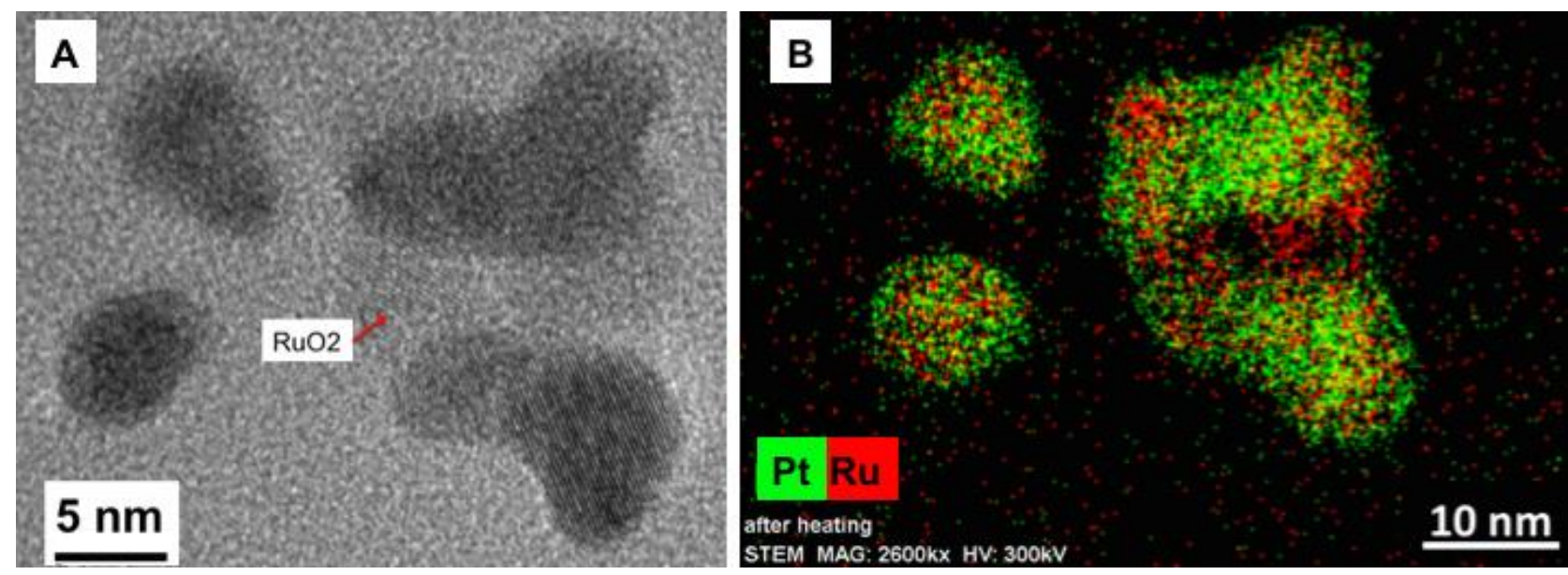

Fig. 2. The last stage of the oxidation of Pt-Ru nanoclusters, showing phase separation of $\mathrm{Ru}$ and formation of $\mathrm{RuO}_{2}$ : (a) BF image of Pt-Ru on SiN support; and (b) EDS mapping of (a) after the in situ heating. 